\title{
A Modular Approach for the Synthesis of Bottlebrush Diblock Copolymers from Poly(Glycidyl Methacrylate)-block-Poly(Vinyldimethylazlactone) Backbones
}

\author{
Bin $\mathrm{Hu}^{1}$, Jan-Michael Carrillo ${ }^{1}$, Liam Collins ${ }^{1}$, Kevin S. Silmore ${ }^{2}$, Jong Keum ${ }^{1}$, Peter V. \\ Bonnesen ${ }^{1}$, Yangyang Wang ${ }^{1}$, Scott Retterer ${ }^{1}$, Rajeev Kumar ${ }^{1, *}$, and Bradley S. Lokitz ${ }^{1, *}$ \\ ${ }^{1}$ Center for Nanophase Materials Sciences, Oak Ridge National Laboratory, Oak Ridge, TN 37831, USA \\ ${ }^{2}$ Chemical Engineering, Massachusetts Institute of Technology, Cambridge, MA 02139, USA
}

\section{Reactivity Tests of PGMA, PVDMA and Hydroxy (-OH), Carboxylic Acid (-COOH) Groups}

Benzyl alcohol and hydrocinnamic acid were used as model compounds to optimize the reaction conditions necessary to independently target reaction with PGMA or PVDMA. For the reaction of PGMA and/or PVDMA with benzyl alcohol, the effect of both catalyst $(1,8$ Diazabicyclo[5.4.0]undec-7-ene, DBU) concentration and reaction temperature were investigated. The initial conditions were based on work by Heilmann and Coworkers who reported the ring opening functionalization of azlactones with alcohols using DBU. ${ }^{1}$ DBU can be used to ring open epoxides for polymerization reactions and/or crosslinking but the reactions are typically done in bulk and/or require elevated temperatures, therefore; it was hypothesized that the catalyst concentration and reaction temperature could be optimized for selective azlactone functionalization. ${ }^{2}$ For the reaction of hydrocinnamic acid with PGMA and/or PVDMA it was postulated that temperature (no catalyst) could be used to drive the reaction carboxylic acids are more nucleophilic than alcohols. The reaction between PGMA and benzyl alcohol or hydrocinnamic acid was monitored using ${ }^{1} \mathrm{H}$ NMR by focusing on the proton peaks associated with epoxy group at 3.8 and $4.3 \mathrm{ppm}$. FTIR was used to monitor the reaction between PVDMA and benzyl alcohol or hydrocinnamic acid, by 
monitoring the $\mathrm{C}=\mathrm{O}$ band from the azlactone peak at $1825 \mathrm{~cm}^{-1}$ (Figure 1). The reactivity results were summarized in Table S1. An X indicates no reaction took place and $\checkmark$ means that measurable conversion took place. The reaction conditions selected to synthesize the bottle brush copolymers that were studied are indicated with a green check mark and red X in the same column of the table.

Table S1. Reactivity results of PGMA and PVDMA with benzyl alcohol or hydrocinnamic acid.

\begin{tabular}{|c|c|c|c|c|c|}
\hline & \multicolumn{5}{|c|}{ Benzyl alcohol $(-\mathrm{OH})$} \\
\hline Catalyst and & 1:1 mol \%, & $10 \mathrm{~mol} \mathrm{\%}$ & 1 mol \% DBU, & $50{ }^{\circ} \mathrm{C}$ & $80{ }^{\circ} \mathrm{C}$ \\
\hline Temperature & $35^{\circ} \mathrm{C}$ & $\mathrm{DBU}, 35^{\circ} \mathrm{C}$ & $35{ }^{\circ} \mathrm{C}$ & & \\
\hline PGMA & $\checkmark$ & $\checkmark$ & $\mathrm{X}$ & $\mathrm{X}$ & $\mathrm{X}$ \\
\hline PVDMA & $\checkmark$ & $\checkmark$ & $\checkmark$ & $X$ & $\checkmark$ \\
\hline
\end{tabular}

\begin{tabular}{|c|c|c|c|c|c|}
\hline & \multicolumn{5}{|c|}{ Hydrocinnamic Acid } \\
\hline Temperature & $50{ }^{\circ} \mathrm{C}$ & $60{ }^{\circ} \mathrm{C}$ & $\mathbf{6 5}^{\circ} \mathbf{C}$ & $70{ }^{\circ} \mathrm{C}$ & $80{ }^{\circ} \mathrm{C}$ \\
\hline PGMA & $\checkmark$ & $\checkmark$ & $\checkmark$ & $\checkmark$ & $\checkmark$ \\
\hline PVDMA & $\mathrm{X}$ & $\mathrm{X}$ & $\mathrm{X}$ & $\checkmark$ & $\checkmark$ \\
\hline
\end{tabular}




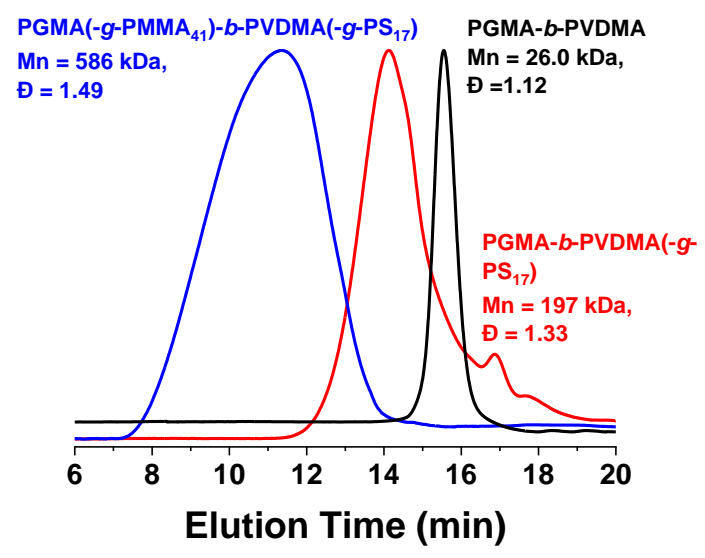

Figure S1. SEC analysis of PGMA- $b$-PVDMA backbone, PGMA- $b$-PVDMA(- $g$-PS 16$)$, and PGMA(- $g$-PMMA48)- $b$-PVDMA(- $g$-PS 16$)$ bottlebrushes.

\section{Bottlebrush molecular weight and grafting density estimations from ${ }^{1} \mathrm{H}-\mathrm{NMR}$}

Molar ratios were determined from the NMR spectra by integrating the peaks assigned to PVDMA (1.4-1.5 ppm), PGMA (3.3 ppm), PS (6.6-7.2 ppm), and PMMA (3.6 ppm). The calculation for calculating molecular weight and estimating grafting density for BB-1 is provided as an example. The molar ratios determined from NMR were normalized so that the PMMA peak had a value of 1 (Table S2). The weight percent of PGMA, PVDMA, PS, or PMMA was calculated by multiplying the mole ratio (determined by NMR) by the molecular weight (determined by SEC) and dividing by the sum of the polymer and grafts (see steps 1-4 below). The MW and dispersity of the parent block copolymer was independently determined by SEC. The MW of the parent block copolymer (numerator) divided by the bottle brush plus grafts (denominator) is equal to the overall weight percent of the parent block copolymer (PGMA- $b$-PVDMA) and allowed the total MW of the parent block copolymer plus grafts to be determined (step 5). The total MW determined 
in step 5 was then multiplied by the weight percent of each graft to determine the combined MW of the PS or PMMA grafts (steps 6-7). The combined MW of PS or PMMA grafts could then be divided by the MW of an individual PS or PMMA chain (independently determined by SEC) to calculate the number of grafts (steps 8-9). The number of PS grafts divided by the number of PVDMA repeat units gives the PS grafting density and the number of PMMA grafts divided by the number of PGMA repeat units gives the PMMA grafting density (steps 10-11).

Table S2: Molar and normalized molar ratios for PVDMA:PGMA, PS:PVDMA, and PMMA:PS

\begin{tabular}{|l|l|l|}
\hline & Mole Ratio & $\frac{\text { Normalized Mole }}{\text { Ratio }}$ \\
\hline PVDMA:PGMA & $1: 0.83$ & $0.08: 0.066$ \\
\hline PS:PVDMA & $1: 0.15$ & $0.53: 0.080$ \\
\hline PMMA:PS & $1: 0.53$ & $1: 0.53$ \\
\hline
\end{tabular}

\section{Sample calculations for determining MW and grafting density in the case of BB-1:}

1. Wt $\% \mathrm{PMMA}=\frac{1(100 \mathrm{~g} / \mathrm{mol})}{1(100 \mathrm{~g} / \mathrm{mol})+0.53(104 \mathrm{~g} / \mathrm{mol})+0.08(139 \mathrm{~g} / \mathrm{mol})+0.066(142 \mathrm{~g} / \mathrm{mol})} \times 100=56.9 \%$

2. Wt $\% \mathrm{PS}=\frac{0.53(104)}{1(100 \mathrm{~g} / \mathrm{mol})+0.53(104 \mathrm{~g} / \mathrm{mol})+0.08(139 \mathrm{~g} / \mathrm{mol})+0.066(142 \mathrm{~g} / \mathrm{mol})} \times 100=31.5 \%$

3. Wt $\%$ PVDMA $=\frac{0.08(139 \mathrm{~g} / \mathrm{mol})}{1(100 \mathrm{~g} / \mathrm{mol})+0.53(104 \mathrm{~g} / \mathrm{mol})+0.08(139 \mathrm{~g} / \mathrm{mol})+0.066(142 \mathrm{~g} / \mathrm{mol})} \times 100=6.3 \%$

4. Wt $\%$ PGMA $=\frac{0.066(142 \mathrm{~g} / \mathrm{mol})}{1(100 \mathrm{~g} / \mathrm{mol})+0.53(104 \mathrm{~g} / \mathrm{mol})+0.08(139 \mathrm{~g} / \mathrm{mol})+0.066(142 \mathrm{~g} / \mathrm{mol})} \times 100=5.3 \%$

5. $\mathrm{MW} \mathrm{BB}-1=\frac{26,000 \frac{\mathrm{g}}{\mathrm{mol}}}{X}=0.116=(P G M A+P V D M A w t \%) / 100=>X=224,138 \mathrm{~g} / \mathrm{mol}$

6. Total PMMA Grafts MW $=0.569 \times 224,138 \mathrm{~g} / \mathrm{mol}=127,535 \mathrm{~g} / \mathrm{mol}$

7. Total PS Grafts MW $=0.315 \times 224,138 \mathrm{~g} / \mathrm{mol}=70,603 \mathrm{~g} / \mathrm{mol}$

8. Number of PMMA Chains Grafted $=\frac{127,535 \mathrm{~g} / \mathrm{mol}}{2,800 \mathrm{~g} / \mathrm{mol}}=46$

9. Number of PS Chains Grafted $=\frac{70,603 \mathrm{~g} / \mathrm{mol}}{2,100 \mathrm{~g} / \mathrm{mol}}=34$

10. PMMA Grafting density $=\frac{46}{72} \times 100=63.9 \%$

11. PS Grafting density $=\frac{34}{111} \times 100=30.6 \%$ 
Table S3. Glass transition temperature $\left(T_{g}\right)$ of PMMA, PS and PGMA- $b$-PVDMA with different DPs.

\begin{tabular}{|c|c|c|c|c|c|c|}
\hline Sample & PMMA $_{24}$ & PMMA $_{48}$ & PS $_{16}$ & PS $_{75}$ & PGMA $_{72-}$ & PGMA $_{338^{-}}$ \\
PVDMA $_{111}$ & PVDMA $_{343}$ \\
\hline$T_{g}\left({ }^{\circ} \mathrm{C}\right)$ & 51.2 & 82.6 & 47.0 & 82.2 & 79.0 & 75.7 \\
\hline
\end{tabular}

Table S4. Backbone DP, $\mathrm{T}_{\mathrm{g}}$, PMMA DP, PS DP, total DP, number of chain ends and fraction of chain ends

\begin{tabular}{|c|c|c|c|c|c|c|c|}
\hline & $\begin{array}{c}\boldsymbol{T}_{\boldsymbol{g}} \\
\left({ }^{\circ} \mathbf{C}\right)\end{array}$ & $\begin{array}{c}\text { Backbone } \\
\mathbf{D P}\left(\mathbf{N}_{\mathbf{b}}\right)\end{array}$ & $\begin{array}{c}\text { Total } \\
\text { PMMA } \\
\text { Graft DP } \\
\left(\mathbf{N}_{\mathbf{g}, \mathbf{P M M A})}\right.\end{array}$ & $\begin{array}{c}\text { Total PS } \\
\text { Graft } \\
\mathbf{D P} \\
\left(\mathbf{N}_{\mathbf{g}, \mathbf{P s})}\right.\end{array}$ & $\begin{array}{c}\text { Total } \\
\mathbf{D P}(\mathbf{N})\end{array}$ & $\begin{array}{c}\text { Number } \\
\text { of chain } \\
\text { ends }\left(\mathbf{n}_{\mathbf{e}}\right)\end{array}$ & $\begin{array}{c}\text { Fraction of } \\
\text { chain ends }\left(\boldsymbol{f}_{\mathbf{e}}\right. \\
\left.=\mathbf{n}_{\mathbf{e}} / \mathbf{N}\right)\end{array}$ \\
\hline BB-1 & 79.4 & 183 & 1104 & 544 & 1831 & 82 & 0.043 \\
\hline BB-2 & 98.1 & 183 & 1920 & 448 & 2551 & 70 & 0.027 \\
\hline BB-3 & 99.0 & 183 & 528 & 600 & 1311 & 32 & 0.024 \\
\hline BB-4 & 116.2 & 183 & 336 & 450 & 969 & 15 & 0.015 \\
\hline BB-5 & 80.9 & 681 & 6720 & 2528 & 9929 & 440 & 0.042 \\
\hline
\end{tabular}




\section{Coarse-Grained Molecular Dynamics Simulation}

Coarse-grained molecular dynamics simulations for different bottlebrush diblock copolymer melts were performed. The chain architecture of the bottlebrush diblock copolymer is described in the main manuscript and a snapshot of the simulation system is shown in Figure 7. The interaction between all beads is described by the Weeks-Chandler-Andersen (WCA) potential,

$$
U_{W C A}(r)=\left\{\begin{array}{cc}
4 \varepsilon_{L J}\left[\left(\frac{\sigma}{r_{i j}}\right)^{12}-\left(\frac{\sigma}{r_{i j}}\right)^{6}-\left(\frac{\sigma}{r_{c u t}}\right)^{12}+\left(\frac{\sigma}{r_{c u t}}\right)^{6}\right] & r<r_{c u t} \\
0 & r \geq r_{c u t}
\end{array}\right.
$$

where $r_{i j}$, is the distance between the $i^{t h}$ and $j^{t h}$ bead, $\varepsilon_{L J}$ is the well depth and $r_{c u t}$ is the cutoff, which is equal to $2^{1 / 6} \sigma$ and $\varepsilon_{L J}=k T$. (Here, $k T$ is the thermal energy and $\sigma$ is the bead diameter). The connectivity of monomers into polymer chains was maintained by the finite extension nonlinear elastic (FENE) potential

$$
U_{F E N E}(r)=-\frac{1}{2} k_{s} R_{m}^{2} \ln \left(1-\frac{r^{2}}{R_{m}^{2}}\right)
$$

with the spring constant $k_{s}=30.0 \mathrm{kT} / \sigma^{2}$, and maximum bond length $R_{m}=1.5 \sigma$. The simulations were carried out using a constant number of particles and temperature ensemble (canonical or $N V T$ ensemble). A constant temperature was maintained by coupling the system to a Langevin thermostat implemented in LAMMPS. ${ }^{3,4}$ The equation of motion for the $i^{\text {th }}$ particle can be described as 


$$
m \frac{d \vec{v}_{l}(t)}{d t}=\vec{F}_{i}(t)-\xi_{L} \vec{v}_{l}(t)+\vec{F}_{i}^{R}(t)
$$

where $\vec{v}_{l}(t)$ is the bead velocity, $\xi_{L}=m / 7 \tau$ is the friction coefficient, and $\vec{F}_{i}(t)$ is the net

deterministic force acting on the bead with mass, $m$ which is set as unity for all beads. $\vec{F}_{i}^{R}(t)$ is the stochastic force with zero average value, $\left\langle\vec{F}_{i}^{R}(t)\right\rangle=0$ and $\delta$-functional correlations $\left\langle\vec{F}_{i}^{R}(t) \vec{F}_{i}^{R}\left(t^{\prime}\right)\right\rangle=6 \xi_{L} k_{B} T \delta\left(t-t^{\prime}\right)$. The velocity-Verlet algorithm with a time step of $0.01 \tau$ was used for time integration. Here, $\tau$ is the characteristic time defined as $\tau=\sigma(m / k T)^{1 / 2}$.

\section{Self-Consistent Field Theory Simulations}

Large-cell self-consistent field theory (SCFT) simulations containing incompressible bottlebrush diblock copolymers were conducted using the PSim software package in two dimensions. ${ }^{5}$ The simulated bottlebrushes consisted of a single backbone with two side chains (i.e., PS and PMMA) spaced equally apart as illustrated in Figure S2. Morphology as a function side chain length was investigated. The backbone is denoted "E", the PS side chain is "S", and the PMMA side chain is "M".

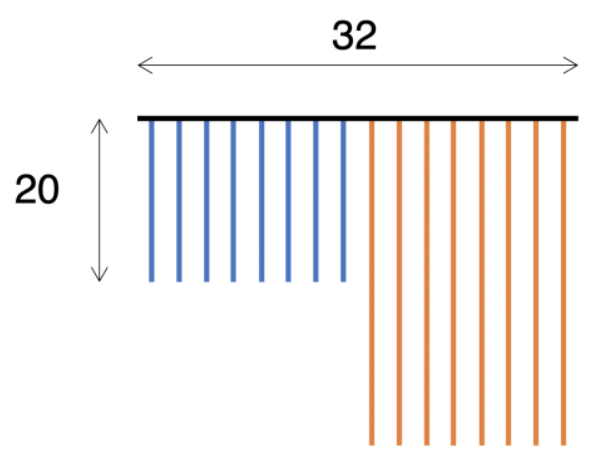

Figure S2: Schematic of bottlebrush components used in SCFT simulations. The backbone is shown in black and the two different side chains (e.g., PS and PMMA) are shown in blue and orange. The total number of Kuhn segments along the backbone was set at 32 - 16 for each block. 
The number of Kuhn segments for the blue side chains was set to 20 while the number of Kuhn segments for the orange side chains was varied.

All of the polymer segments were assumed to have the same length, $l$, and lengths were nondimensionalized by the quantity $l \sqrt{N / 6}$ (the radius of gyration of a freely jointed, linear chain of length $N$ ), where $N=\sum_{i \in\{E, M, S\}} N_{i}$ is the sum of all components' segment lengths in each bottlebrush. In real units, $l$ was estimated as $l=v^{1 / 3}=5.3 \AA$, where $v$ is the harmonic average of the molar volumes of PS and PMMA, which in turn were estimated using bulk density values of $1.14 \mathrm{~g} / \mathrm{cm}^{3}$ and $1.20 \mathrm{~g} / \mathrm{cm}^{3}$, respectively. ${ }^{6}$ Three independent Flory-Huggins interaction parameters, $\chi_{S M}, \chi_{S E}$, and $\chi_{M E}$, were used to describe interactions between the different side chain components and between the side chains and the backbone. To account for incommensurability box size, simulations were conducted on square grids ranging from $48 \times 48$ nodes to $80 \times 80$ nodes, and the grid associated with the lowest free energy was selected.

Figure $\mathbf{S 3}$ shows the morphologies as a function of side chain length at values of $\chi N$ at or above the order-disorder transition (Figure 8) as a function of side chain segments, $f_{M} /\left(f_{S}+f_{M}\right) . f_{i=M, S}$ is defined as the overall fraction of segments in each bottlebrush block copolymer of given type. Figure S4 is the partial structure factors determined for each component from the morphologies shown in Figure S3 (calculated via a fast Fourier transform). The partial structures are not as smooth as those calculated from experimental scattering data due to the periodicity of the simulations. Figures S3 and S4 suggest that the bottle deblock copolymers transition from lamellae to hexagonal close-packed cylinders when the length of one side chain is increased which is similar to what would be expected for linear diblock copolymers. ${ }^{7-10}$ Furthermore, Figure $\mathbf{S 3}$ indicates that the backbone is predominantly located at the interface between the $\mathrm{S}$ and $\mathrm{M}$ microphases. 


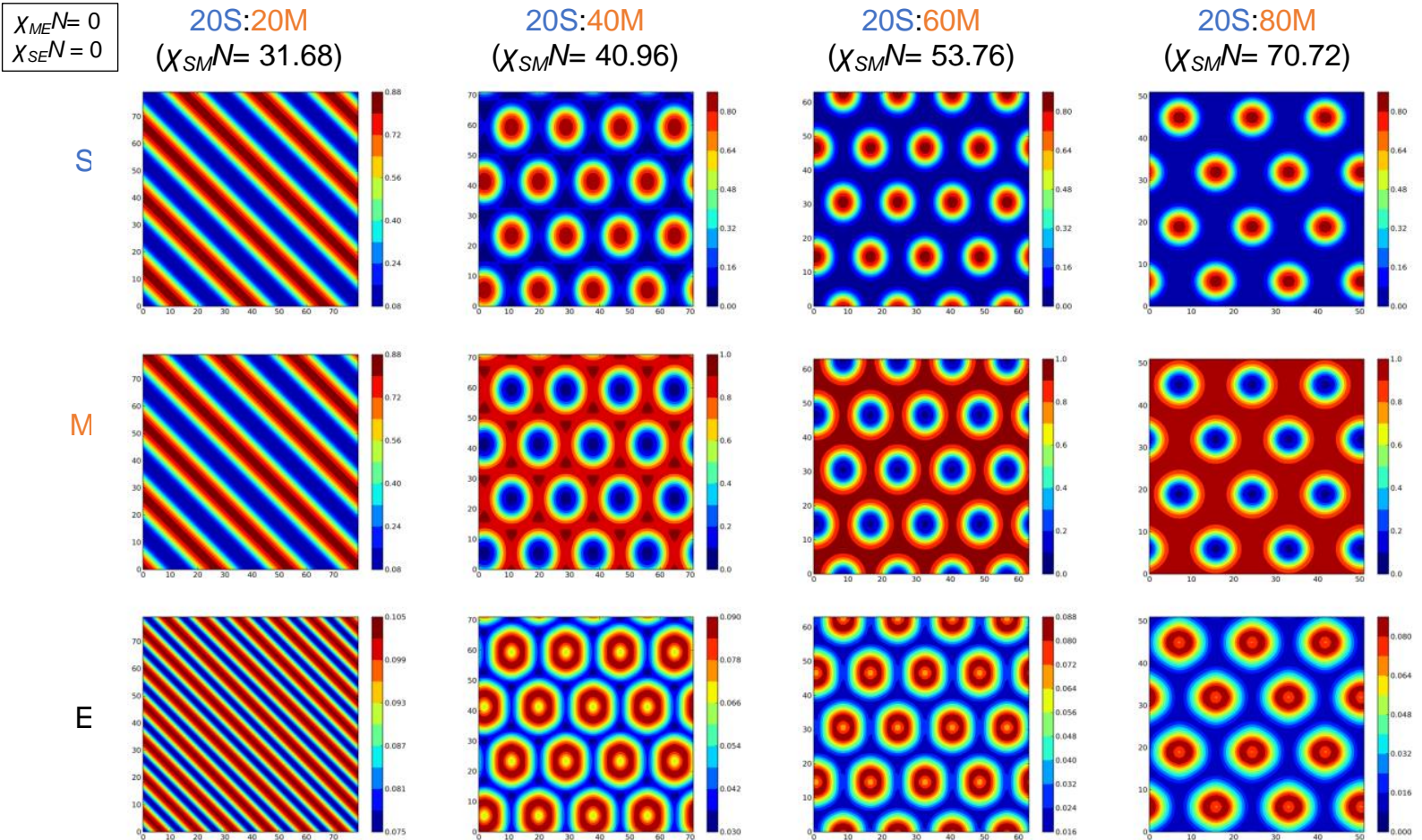

Figure S3: Bottlebrush morphologies as a function of graft ratio (S:M). Rows S, M, and E are the volume fractions of the individual component ( $\mathrm{S}, \mathrm{M}$ or $\mathrm{E})$. Interaction parameters were set to $\chi_{M E}=\chi_{S E}=0$. 


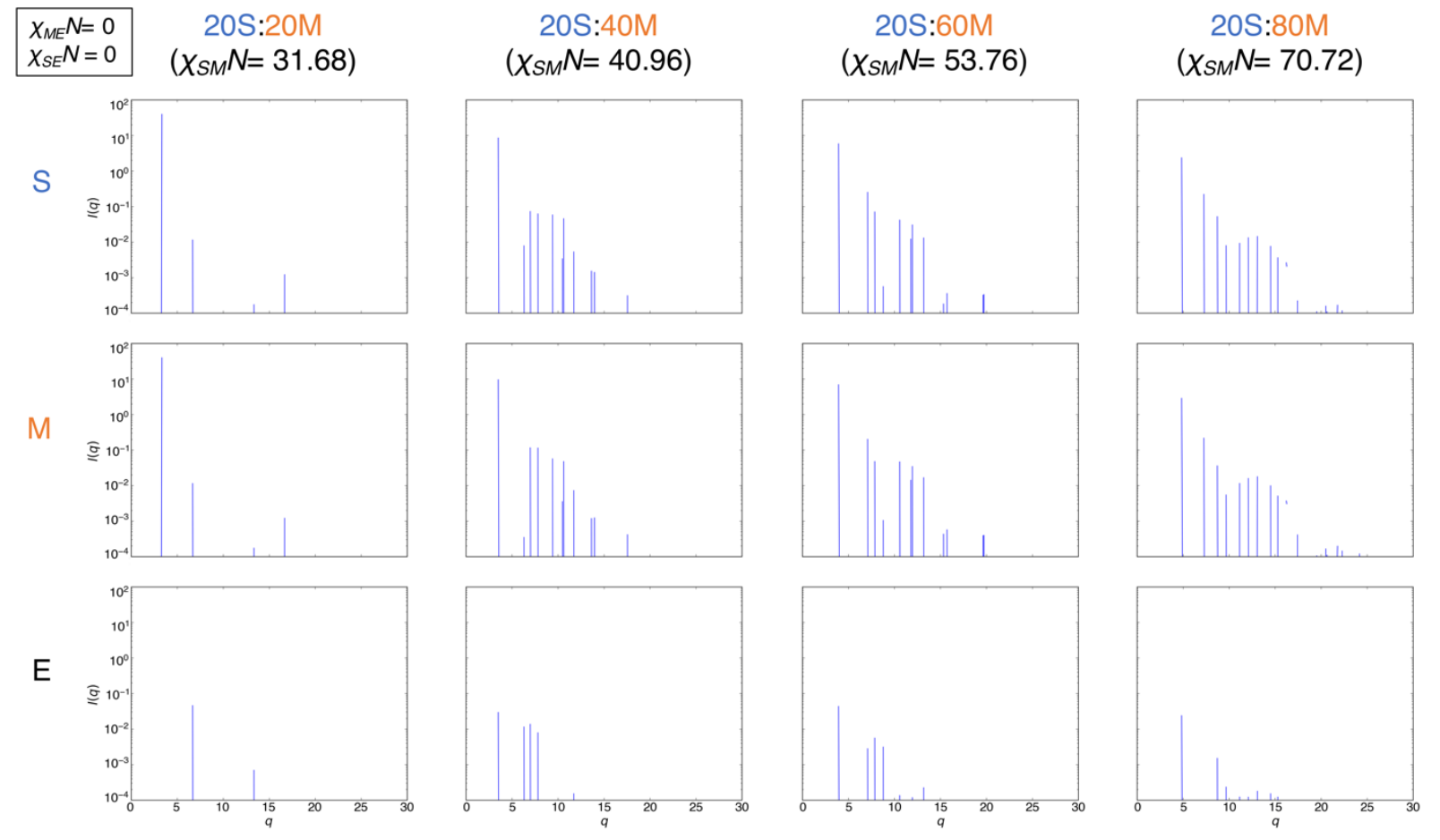

Figure S4: Partial structure factors for various relative graft lengths (corresponding to those in Figure S3). Wavevectors are in units of inverse length and were made dimensionless via the associated freely jointed radius of gyration, as discussed in the text.

\section{References}

1. Heilmann, S. M; Moren, D. M.; Krepski, L. R.; Pathre, S. V.; Rasmussen, J. K.; Stevens, J., The Chemistry of 2-Alkenyl-5(4H)-Oxazolones. VIII Acid-Catalyzed Reaction with Alcohols. Tetrahedron, 1998, 54, 12151-12160.

2. Kudo, H.; Buya, K., Mechanistic Study of Ring-Opening Copolymerization of -Caprolactam with Epoxide: Development of Novel Thermosetting Epoxy Resin System. J. Polym. Sci., Part A: Polym. Chem. 2016, 54, 2220-2228.

3. Plimpton, S., Fast Parallel Algorithms for Short-Range Molecular Dynamics. J. Comput. Physics 1995, 117 (1), 1-19. 
4. Brown, W. M.; Wang, P.; Plimpton, S. J.; Tharrington, A. N., Implementing molecular dynamics on hybrid high performance computers - short range forces. Comput. Phys. Commun. 2011, 182 (4), 898-911.

5. Sides, S. W.; Fredrickson, G. H., Parallel algorithm for numerical self-consistent field theory simulations of block copolymer structure. Polymer 2003, 44 (19), 5859-5866.

6. Kim, H.; Arras, M. M. L.; Mahalik, J. P.; Wang, W.; Yu, D. M.; Chernyy, S.; Goswami, M.; Kumar, R.; Sumpter, B. G.; Hong, K.; Smith, G. S.; Russell, T. P., Studies on the 3-Lamellar Morphology of Miktoarm Terpolymers. Macromolecules 2018, 51 (19), 7491-7499.

7. Matsen, M. W.; Bates, F. S., Unifying Weak- and Strong-Segregation Block Copolymer Theories. Macromolecules 1996, 29 (4), 1091-1098.

8. Jiang, L.; Nykypanchuk, D.; Pastore, V. J.; Rzayev, J., Morphological Behavior of Compositionally Gradient Polystyrene-Polylactide Bottlebrush Copolymers. Macromolecules 2019, 52 (21), 8217-8226.

9. Gai, Y.; Song, D.-P.; Yavitt, B. M.; Watkins, J. J., Polystyrene-block-poly(ethylene oxide) Bottlebrush Block Copolymer Morphology Transitions: Influence of Side Chain Length and Volume Fraction. Macromolecules 2017, 50 (4), 1503-1511.

10. Zhulina, E. B.; Sheiko, S. S.; Dobrynin, A. V.; Borisov, O. V., Microphase Segregation in the Melts of Bottlebrush Block Copolymers. Macromolecules 2020, 53 (7), 2582-2593. 